\title{
LES PUBLICS EUROPÉENS DU JOURNAL TÉLÉVISÉ
}

\author{
François Heinderyckx ${ }^{1}$
}

L'omniprésence du discours annonçant ou observant divers mouvements de convergence économique, politique, culturelle et sociale en Europe voire dans le monde pourrait laisser croire, un peu précipitamment, qu'il s'agit d'une tendance inéluctable et universelle. Globalisation, convergence, mondialisation, synchronisation, impérialisme culturel, autant de concepts et théories dont l'utilisation souvent en dehors de leur contexte original se révèle propice à la dénaturation et au galvaudage qui conduisent, au mieux, à des simplifications outrancières; au pire à des contresens ou des mystifications.

\section{Information transnationale}

Le domaine des médias d'information constitue un terrain fertile pour ce genre de malentendu, en raison de la coüncidence récente entre une restructuration du secteur et une révolutions technique, à savoir entre de violents mouvements de concentration des entreprises contrôlant les différents échelons de l'information médiatisée et

1 Faculté de philosophie et lettres, Section de communication, information et journalisme de l'Université Libre de Bruxelles. 
l'avènement des technologies de télécommunication permettant la diffusion véritablement transnationale de supports médiatiques. Curieusement, on ne peut déceler de lien de cause à effet entre ces deux phénomènes : l'innovation technique ne saurait expliquer la concentration; cette dernière n'a pas, à elle seule, suscité la première. Mais la juxtaposition des deux a réuni les conditions nécessaires à l'apparition d'un nouveau type de support médiatique, le média transnational ${ }^{1}$, engendrant dans son sillage quelques initiatives, encore isolées mais néanmoins persévérantes, de ce qu'on qualifie communément de médias transnationaux d'information.

Cable News Network (CNN), pionnier et figure emblématique du genre, n'est que l'arbre qui cache la forêt, puisque bien d'autres initiatives ont vu le jour tant en télévision qu'en presse écrite et en radio. Ce dernier média a d'ailleurs connu des exemples précoces de supports transnationaux : $B B C$ World Service, Deutsche Welle ou Radio France International exploitaient les premières technologies de diffusion hertzienne à longue portée bien avant que ne germe le concept CNN dans l'esprit de Ted Turner.

C'est pourtant CNN qui demeure la référence, l'étalon auquel se mesurent la plupart des aventures de supports d'actualité à diffusion transnationale, à plus forte raison lorsqu'il s'agit de télévision. Le secteur public n'est pas en reste dans ce tumulte de projets, à tel point qu'une association dénommée le Groupe de Bruges, réunissant pas moins de douze «chaînes satellitaires transnationales européennes de service public $»^{2}$ a été fondée en 1991. Parmi celles-ci, Euronews mérite une mention spéciale tant elle constitue la plus audacieuse initiative de média transnational d'information en Europe ${ }^{3}$. Les motivations qui ont conduit à la création d'Euronews et qui justifient la priorité accordée à sa survie, peuvent être regroupées autour de trois pôles : briser le monopole américain de l'information transnationale en continu (l'omniprésence de $C N N$ pendant la guerre du Golfe

1 Nous entendons par médias transnationaux ceux diffusant explicitement et délibérément à l'intention d'un ou de plusieurs publics répartis sur une aire géographique transcendant les frontières nationales.

2 Les chaînes membres de ce groupe sont Arte, BBC TV World Ltd, RAISAT, Deutsche Welle TV, RTVE Int. (Espagne), TRT Int. (Turquie), Euronews, RTP Int. (Portugal), 3 SAT, TV5, DUNA TV (Hongrie) et TVP-TV Polonia (Pologne). Notons que ces chaines ne sont pas toutes transnationales au sens strict de notre définition.

3 Euronews est née en 1993 sur les cendres d'Europa TV (expérience avortée - 1987 de chaine d'information par satellite). 
constitua, de ce point de vue, un électrochoc ${ }^{1}$ ); assurer la présence du service public dans ce genre encore récent et tellement stratégique (Euronews est le fruit de la volonté commune des télévisions publiques française, italienne, suisse et espagnole, avec le soutien de l'UER); et enfin contribuer activement à promouvoir une opinion publique européenne et participer ainsi à la construction européenne (une télévision continentale constitue un vieux rêve des autorités communautaires).

Précurseurs des médias à venir pour les optimistes et les inquiets; expériences anecdotiques sans lendemain pour les sceptiques; les supports transnationaux ne peuvent laisser indifférent. Le scepticisme se nourrit essentiellement du fait que bien des obstacles jalonnent la route de ce genre de projet, au premier rang desquelles figure un amas d'écueils commodément réunis sous l'appellation nébuleuse de "diversité culturelle". Indiscutablement, cette pluralité multidimensionnelle se révèle potentiellement rédhibitoire pour toute diffusion médiatique d'envergure internationale, tant les produits médiatiques sont manifestement empreints d'une "texture sociodémographique"2, sorte de saveur confuse mais largement perceptible dont les relents évoquent d'une manière ou d'une autre l'environnement de production.

Dans ce contexte, nous avons entrepris d'étudier la fréquence de consommation des journaux télévisés dans les douze pays d'Europe occidentale qui constituaient la Communauté européenne jusqu'à son dernier élargissement. Une étude comparative sur une telle échelle se heurte généralement à l'hétérogénéité des données disponibles, essentiellement en raison de la diversité, dans les différents pays, des méthodologies de collecte, des critères retenus et même de la définition même du comportement étudié. Nos recherches nous ont menées à la Commission européenne dont certaines enquêtes présentent des garanties satisfaisantes d'homogénéité méthodologiques. Plus particulièrement, la Direction Générale X organise périodiquement des

1 Remarquons, à ce propos, que la Guerre du Golfe a été créditée de tous les maux et de tous les changements intervenus depuis son déroulement. Jochen Zimmer relève, en particulier, que le lancement de nombreuses chaînes d'information en continu a été soi-disant suscité par ce conflit alors qu'il est plutôt le résultat des progrès technologiques, de la dérégulation, etc. (J. ZIMMER, "Ware nachrichten", in Media Perspektiven, $\mathrm{n}^{\circ}$ 6/93, Francfort-sur-Main, ARD-Werbegesellschaften, 1993, p. 278-289).

2 Pour reprendre l'expression de M. BASSAND, Culture et régions d'Europe, Lausanne, Presses Polytechniques et Universitaires Romandes, 1990, p. 151. 
sondages d'opinion à l'échelle européenne dont les résultats sont publiés sous l'appellation d'Eurobaromètre. En dépit de la grande variété des sujets abordés, un certain nombre de thèmes sont étudiés de façon récurrente et, parmi ceux-ci, la fréquence de consommation des journaux télévisés, des journaux parlés et de la presse quotidienne. Après diverses opérations d'agrégation (notamment de quatre sondages successifs ${ }^{1}$ ), nous disposons de distributions d'effectifs de "gros consommateurs"2 de journaux télévisés suivant diverses ventilations sociodémographiques.

\section{Douze nations, un continent}

Sur cette base, il apparaît que 89 pour cent des Européens regardent un journal télévisé au moins plusieurs fois par semaine (notre définition des "gros consommateurs"). Les données varient d'un pays à l'autre, les extrêmes étant occupés par la France et le Portugal d'une part (82 pour cent), l'Italie d'autre part (94 pour cent). Le Graphique 1 détaille des valeurs ventilées par pays.

Ce faible étalement (coefficient de variation : 5 pour cent) indique une propension importante des Européens à s'informer par le média télévisuel.

Il est difficile de recouper ces chiffres avec les scores d'audience des journaux télévisés, d'abord parce que ceux-ci sont programmés sur des chaînes concurrentes à des heures parfois différentes et il est, de ce fait, impossible d'estimer le taux de redondance, à savoir le nombre d'individus qui regardent successivement plusieurs journaux télévisés. Ainsi, en Belgique francophone, les journaux de 19 heures sur $R T L-T V i$ et de 19 heures 30 sur la $R T B F$, font état d'une audience d'environ 20 pour cent, sans qu'il soit possible d'additionner ces deux valeurs sachant que nombre de téléspectateurs regardent successivement l'un et l'autre, sans parler de ceux qui regardent un journal télévisé de la mi-journée ou de fin de soirée, ou d'une chaine néerlandophone ou étrangère.

1 Vagues 38, 39, 40 et 41 menées de 1992 à 1994 à partir d'un total cumulé de 52.225 interviews.

2 La classe que nous avons intitulée "Gros consommateur" résulte de l'agrégation des effectifs d'individus déclarant regarder le journal télévisé "tous les jours" ou "plusieurs fois par semaine". 


\section{Graphique 1}

Proportions de gros consommateurs de journaux télévisés

(ventilées par pays).

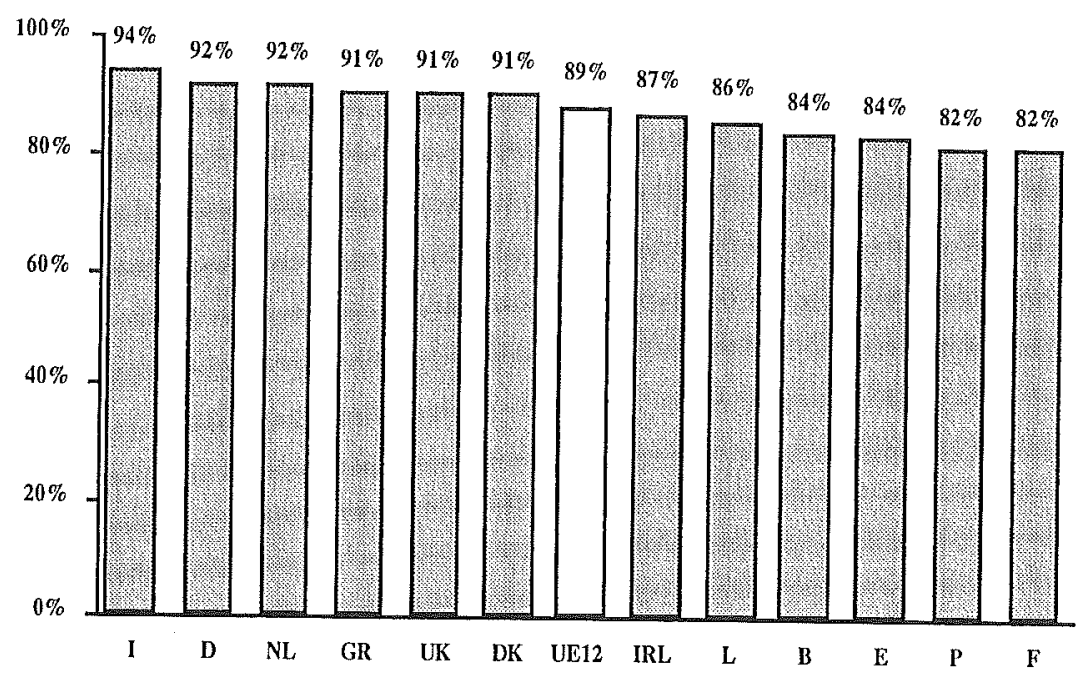

A partir de notre agrégation des sondages $38,39,40$ et 41 de l'Eurobaromètre. Coefficient de variation : $5 \%$.

Remarquons que ce type d'émission occupe la place enviée de programme le plus suivi sur les chaines de Belgique francophone $(R T L-T V i$ et $R T B F)$. Ce "doublé" est unique en Europe'. D'autres journaux télévisés affichent des scores d'audience supérieurs à ceux observés en Belgique francophone, mais toujours battus, au palmarès de plus fortes audiences, par d'autres programmes de la même chaîne (fictions, jeux, etc.). Les scores cumulés des journaux de $T F l$ et France 2 (on peut les additionner puisqu'ils sont programmés à la même heure) atteignent les 30 pour cent, le 20 heures 30 de YLEl (Finlande) dépasse les 45 pour cent ${ }^{2}$.

1 Seules la danoise DR et la finlandaise YLEl et la norvégienne NRK affichent également en tête de leur palmarès le journal télévisé de début de soirée, mais sans que pareil phénomène soit observé sur les autres chaînes généralistes de ces pays (Chiffres pour 1994 dans European key facts - Television 95 publié par IP). Ce succès en Norvège est d'ailleurs évoqué par I. HAGEN, "Expectations and consumption patterns in television news viewing", in Medias, Culture and Society, vol.16, n³, Londres, Sage, 1994, p. 425.

2 Les données s'entendent pour les pays respectifs des chaînes citées. Chiffres pour 1994 dans European key facts - Television 95 publié par IP. 
Notons encore que le temps passé devant le téléviseur n'est aucunement corrélé avec la consommation de journaux télévisés telle que nous l'envisageons. A titre d'exemple, les chiffres publiés par Médiamétrie/Eurodata présentent les Portugais comme les plus gros consommateurs de télévision (258 minutes par jour ${ }^{1}$ ) alors que, selon l'Eurobaromètre, ils sont parmi les moins friands de journaux télévisés (voir Tableau 1). Il semble ainsi démontré qu'on ne peut ramener la problématique de la consommation de médias d'information à une simple question de "média préféré". Il aurait pourtant été séduisant de penser que telle population consommant plus de tel média serait nécessairement portée à s'informer par ce biais.

\section{Tableau 1}

Consommation de journaux télévisés

et consommation quotidienne de télévision

\begin{tabular}{lccc}
\hline & $\begin{array}{c}\text { Gros } \\
\text { consommateurs } \\
\text { journaux télévisés } \\
(1)\end{array}$ & $\begin{array}{c}\text { Durée moyenne } \\
\text { devant la télévision }\end{array}$ & $\begin{array}{c}\text { Passent plus de } \\
\text { 2h30 devant la } \\
\text { télévision (3) }\end{array}$ \\
\hline I & $94 \%$ & $207 \mathrm{~min}$ & $28 \%$ \\
D & $92 \%$ & $178 \mathrm{~min}$ & $50 \%$ \\
NL & $92 \%$ & $147 \mathrm{~min}$ & $45 \%$ \\
GR & $91 \%$ & $183 \mathrm{~min}$ & $44 \%$ \\
UK & $91 \%$ & $220 \mathrm{~min}$ & $56 \%$ \\
DK & $91 \%$ & $147 \mathrm{~min}$ & $38 \%$ \\
IRL & $87 \%$ & $191 \mathrm{~min}$ & $48 \%$ \\
L & $86 \%$ & - & - \\
B & $84 \%$ & $144 \mathrm{~min}$ & $45 \%$ \\
E & $84 \%$ & $204 \mathrm{~min}$ & $39 \%$ \\
P & $82 \%$ & $258 \mathrm{~min}$ & - \\
F & $82 \%$ & $177 \mathrm{~min}$ & $50 \%$ \\
\hline
\end{tabular}

(1) Selon notre agrégation des sondages 38, 39, 40 et 41 de l'Eurobaromètre.

(2) Par jour, selon Médiamétrie / Eurodata TV, évoqué par Christine Simon, "Les téléspectateurs sont fous de foot", Le Soir, 22 avril 1994, p. 17.

(3) INRA Global Survey 1995.

1 Chiffres cités par C. Simon, "Les téléspectateurs sont fous de foot", in Le Soir, 22 avril 1994, p. 17. 
Remarquons d'autre part que les chiffres fournis par une troisième enquête, celle effectuée en 1995 par l'INRA', évaluant la proportion d'individus déclarant regarder la télévision plus de deux heures et demi par jour ne concordent pas non plus avec les proportions de gros consommateurs de journaux télévisés, pas plus, d'ailleurs, qu'avec les données de Médiamétrie/Eurodata.

La consommation de journaux télévisés peut également être confrontée aux résultats d'un autre sondage étudiant (entre autres choses) la proportion d'individus désirant davantage d'émissions d'information (voir Graphique 2).

\section{Graphique 2}

Proportions d'individus désirant davantage d'émissions d'information en fonction des proportions de gros consommateurs de journaux télévisés.

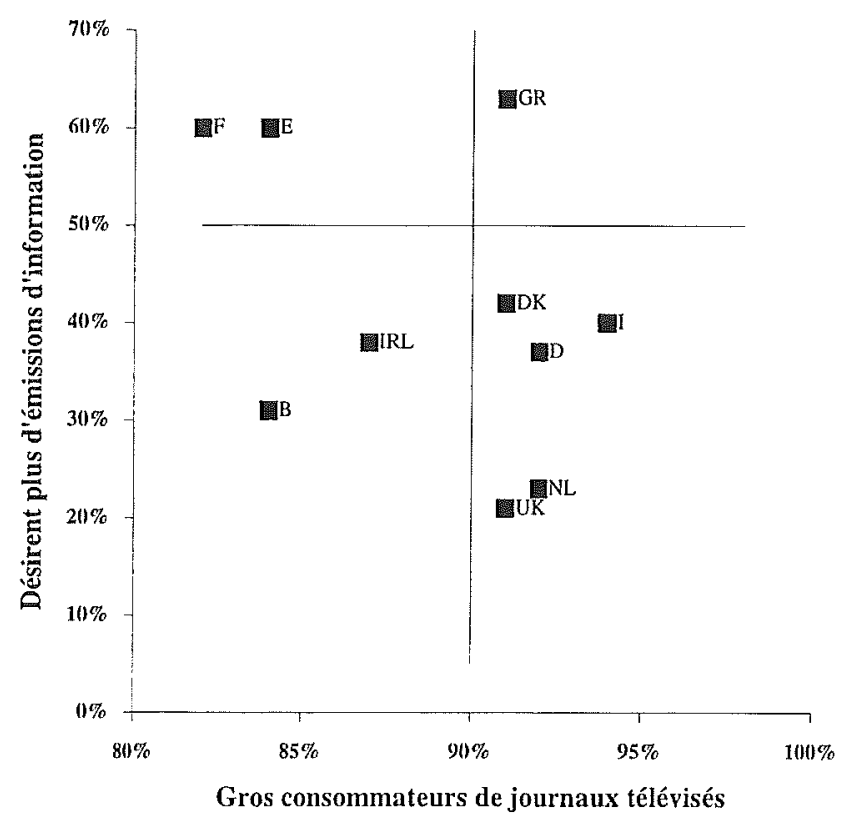

Source : notre agrégation des sondages $38,39,40$ et 41 de l'Eurobaromètre et INRA, Global Survey 1995.

1 INRA Global Survey, enquête effectuée dans 43 pays du monde pour le $50^{\circ}$ anniversaire de l'INRA. Résultats partiels publiés dans Citizens of the World, Consumers of the World; données complètes obtenues auprès de l'INRA. 
Les données considérées par pays ${ }^{1}$ peuvent être réparties en quatre groupes matérialisés sur notre graphique par deux axes perpendiculaires définissant quatre quadrants. Allemagne, Danemark, Italie, Pays-Bas et Royaume-Uni présentent des proportions importantes de gros consommateurs de journaux télévisés, et une faible proportion d'individus désirant davantage d'émissions d'information, à l'inverse des Français et des Espagnols qui sont moins assidus mais en aimeraient plus. Belges et Irlandais comptent moins de gros consommateurs, mais semblent se satisfaire de l'offre, alors que les Grecs présentent d'importantes proportions de gros consommateurs et d'insatisfaits (qui en voudraient davantage).

Pour en revenir aux chiffres de l'Eurobaromètre, on ne constate pas de différence significative entre hommes et femmes, pas plus qu'entre professions, classes sociales ou types d'habitat. Le comportement n'est pas pour autant universel. Ainsi, les revenus semblent intervenir dans certains pays, mais le facteur le plus déterminant semble être 1'âge. Nous disposons des données pour quatre classes d'âge (voir Tableau 2).

Tableau 2

Proportions de gros consommateurs de journaux télévisés, par âge (en pour cent)

\begin{tabular}{cc}
\hline Tranches d'âge & UE12 \\
\hline 15-24 ans & 81 \\
$25-39$ ans & 88 \\
40-54 ans & 91 \\
55 ans et plus & 93 \\
\hline
\end{tabular}

A partir de notre agrégation des sondages 38, 39, 40 et 41 de l'Eurobaromètre.

Cette tendance se retrouve dans presque tous les pays, à l'exception du Portugal (qui tombe à 78 pour cent pour les 55 ans et plus contre 81 ou 86 pour cent pour le reste de la population). Le phénomène est particulièrement spectaculaire en Belgique, au Danemark, en France, en Irlande et au Luxembourg, où l'écart est de près de 20

1 Le Luxembourg et le Portugal n'étant pas inclus dans l'étude de l'INRA ne peuvent être pris en compte. 
points de pour cent entre les 15-24 ans et les 55 ans et plus. L'Espagne, l'Italie, les Pays-Bas et la Grèce se distinguent, à l'inverse, par un faible écart. Remarquons que les valeurs culminent à 96 voire 97 pour cent pour les 55 ans et plus habitant le Danemark, le Royaume-Uni et l'Allemagne.

\section{Nations, régions}

L'approche comparative s'inscrit trop souvent exclusivement dans une perspective centrée autour des Etats-nations. Bien des facteurs concourent à la pérennité de cette vision restrictive : organisation de la collecte des données alimentant les fonds statistiques, données émanant des médias (eux-mêmes structurés suivant les nations), processus de sélection des informations organisé autour de l'identité nationale, particularités législatives et réglementaires.

Nous allons nous employer à envisager les agencements alternatifs, à commencer par d'autres divisions territoriales, avant d'examiner des découpages transversaux, notamment suivant quelques critères sociodémographiques, transcendant les frontières et la perspective géographique en général.

Quand on songe à dépasser la perspective nationale, c'est généralement dans une démarche d'arborescence simple, du national vers le régional, au sens subnational. Cette dernière approche a le mérite de permettre de prendre la mesure des écarts entre communautés culturelles reconnues voire institutionnalisées (notamment dans les États fédéraux), mais aussi de faire apparaître l'hétérogénéité parfois marquée présente dans certains pays pourtant perçus, a priori, comme assez homogènes. En l'occurrence, il s'agit d'aller plus loin, en envisageant les régions comme entités subeuropéennes, en tentant de faire abstraction de toute appartenance nationale et en cherchant à révéler d'éventuels ensembles multirégionaux et transnationaux.

Les données de l'Eurobaromètre sont disponibles suivant une ventilation régionale que nous avons agrégé en 60 unités territoriales de tailles et d'effectifs comparables. La Carte 1 permet de visualiser la densité des différentes régions en gros consommateurs de journaux télévisés. Trois remarques s'imposent afin d'éclairer la lecture de cette carte. Premièrement, les frontières nationales ont été délibérément omises sur cette carte afin de ne pas biaiser la perception d'éventuelles zones régionales transnationales. Il ne faut cependant 
pas perdre de vue la physionomie du découpage (apparaissant en blanc sur nos cartes) afin d'éviter d'être leurré par d'éventuelles homogénéités "techniques" qui seraient attribuables au découpage agrégé. Ainsi, par exemple, il ne serait pas opportun d'observer une grande homogénéité en Irlande, au Danemark ou au Luxembourg, ces trois pays ne faisant pas l'objet, pour des raisons techniques, de découpages en régions dans notre agrégation. Deuxièmement, il convient de garder à l'esprit que les écarts sont, dans l'absolu, assez faibles et que, dès lors, les cinq classes de valeurs s'en trouvent exiguës. Enfin, la carte a été calibrée afin que la moyenne européenne pondérée se situe dans la catégorie médiane des classes de valeurs. De cette manière, chaque zone est d'emblée située au-dessus, en dessous ou au niveau de la moyenne européenne.

On perçoit d'abord un partage assez tranché de l'Europe entre, d'une part, la majeure partie de l'Allemagne (à l'exclusion de la Saxe), les Pays-Bas, le Royaume-Uni, l'Italie et la Grèce (sauf la Crète) qui se situent à la moyenne ou au-dessus et, d'autre part, le reste de l'Europe continentale qui, à l'exception du nord de l'Espagne, présente des valeurs inférieures à la moyenne. 


\section{Carte 1}

Proportions de gros consommateurs de journaux télévisés

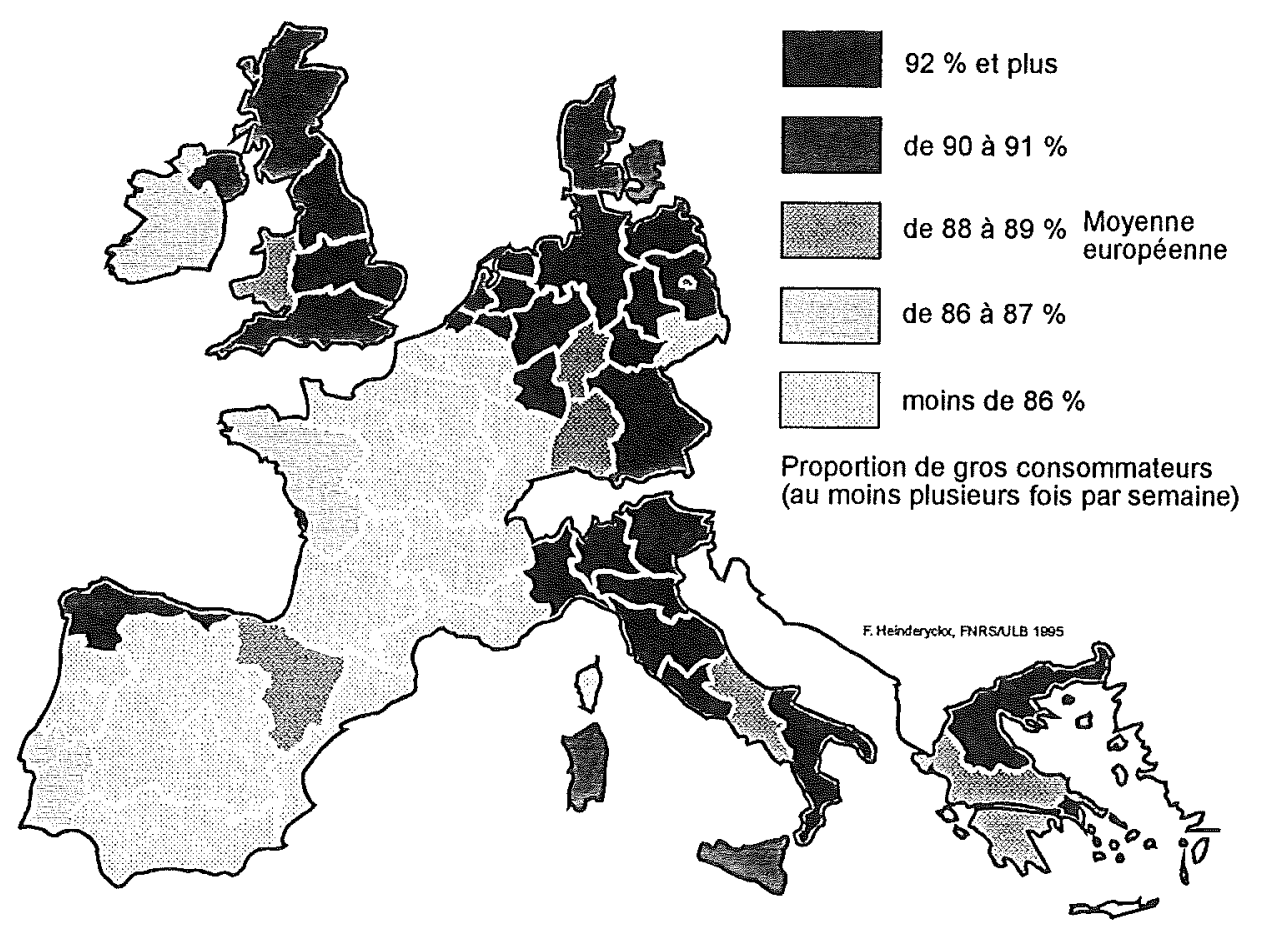

Carte réalisée sur base de notre agrégation des vagues $38,39,40$ et 41 de leurobaromètre 
Relevons le fait que les valeurs considérées par régions (étalement entre les 60 régions) apparaissent plus dispersées que lorsque nous les avions envisagées par pays (voir Tableau 3).

Tableau 3

Comparaison des étalements entre pays et entre régions (en pour cent)

\begin{tabular}{lccc}
\hline & Minimum & Maximum & $\begin{array}{c}\text { Coefficient de } \\
\text { variation }\end{array}$ \\
\hline Entre pays & 82 & 94 & $4,5 \%$ \\
Entre régions & 74 & 97 & $6,0 \%$ \\
\hline
\end{tabular}

A partir de notre agrégation des sondages $38,39,40$ et 41 de l'Eurobaromètre.

Ce constat semble indiquer que les valeurs appréhendées à l'échelle des nations atténuent et donc dissimulent des variances subnationales. Le Tableau 4 indique, pour chaque pays, le coefficient de variation de la série constituée des valeurs pour ses différentes régions ${ }^{1}$.

Tableau 4

Coefficients de variation des valeurs ventilées par régions (en pour cent)

\begin{tabular}{lcc}
\hline & $\begin{array}{c}\text { Moyenne } \\
\text { nationale }\end{array}$ & $\begin{array}{l}\text { C. variat } \\
\text { interrég'le }\end{array}$ \\
\hline Allemagne & 92 & $6,0 \%$ \\
Belgique & 84 & $1,0 \%$ \\
Espagne & 84 & $3,9 \%$ \\
France & 82 & $2,3 \%$ \\
Grèce & 91 & $4,8 \%$ \\
Italie & 94 & $2,6 \%$ \\
Pays-Bas & 92 & $1,4 \%$ \\
Portugal & 82 & $5,0 \%$ \\
Royaume-Uni & 91 & $2,1 \%$ \\
\hline Moyenne & 88 & $3,2 \%$ \\
Coef. variation & $5,2 \%$ & \\
\hline
\end{tabular}

A partir de notre agrégation des sondages 38, 39, 40 et 41 de l'Etrobaromètre.

1 Sont exclus de ce calcul le Danemark, le Luxembourg et l'Irlande, pour lesquels l'agencement de nos données ne présente pas de découpage subnational. 
Une série de constatations s'imposent à la lecture de ces chiffres. Rappelons que les coefficients de variation sont directement comparables ${ }^{1}$. Les valeurs présentées sur le Tableau 4 renforcent le constat du moindre étalement des données des journaux télévisés, puisque le coefficient de variation moyen n'est que de 3,2 pour cent.

D'importants écarts de diversité subnationale sont à constater entre les neuf pays envisagés dans ce volet. Le Portugal, la Grèce et surtout l'Allemagne présentent les valeurs les plus hétérogènes. L'Allemagne affiche même un coefficient de variation ( 6 pour cent) supérieur à celui calculé sur les moyennes nationales des neuf pays considérés (5,2 pour cent). Autrement dit, les douze régions allemandes ne seraient pas plus homogènes, du point de vue de la consommation de journaux télévisés, que l'Europe entière. $\mathrm{Ne}$ perdons pas de vue que l'Allemagne compte, dans notre étude, le plus grand nombre de régions. La Belgique et les Pays-Bas semblent les plus homogènes de ce point de vue mais, à l'inverse, comptent un nombre réduit de régions.

\section{Groupes transversaux}

La recherche d'éventuels groupes transversaux qui transcenderaient l'appartenance nationale passe par la comparaison des indices de dispersion (ici le coefficient de variation) entre les différentes classes des critères sociodémographiques considérés. Ainsi, nous comparerons la dispersion entre hommes et femmes à celle entre les quatre classes d'âge, les trois types d'habitat, etc.

Comment interpréter ces données ? Plus l'indice d'étalement des données est élevé, plus il indique des différences importantes entre les catégories du critère envisagé, et donc plus ce critère peut-être considéré comme différenciateur du comportement étudié. Illustrons par l'exemple.

Le Tableau 5 montre que l'âge et la profession présentent les indices de dispersion les plus élevés. Cela signifie qu' on peut observer des concentrations très diverses de gros consommateurs de

1 Le coefficient de variation (quotient de l'écart type par la moyenne) présente l'avantage d'inclure la moyenne dans la mesure d'étalement. Ainsi, ce coefficient est sans unité (généralement exprimée en pour cent) et permet de comparer directement l'étalement de séries, même si celles-ci ne sont pas du même ordre de grandeur ou si elles ne sont pas exprimées dans la même unité. 
journaux télévisés selon la tranche d'âge et selon la profession. A l'inverse, le sexe et l'habitat sont sans effet sur ce comportement, c'est-à-dire que les valeurs varient très peu entre hommes et femmes ou entre habitat rural et urbain.

Tableau 5

Etalement des données entre catégories des différents critères sociodémographiques (coefficients de variation, en pour cent)

\begin{tabular}{lccccccc}
\hline & Sexe & Age & Revenus & Habitat & $\begin{array}{c}\text { Profes- } \\
\text { sion }\end{array}$ & $\begin{array}{c}\text { Classe } \\
\text { sociale }\end{array}$ & Région \\
\hline $\mathrm{D}$ & 0,9 & 5,4 & 0,4 & 0,5 & 1,6 & 0,9 & 6,0 \\
$\mathrm{~B}$ & 3,2 & 9,1 & 1,0 & 0,8 & 5,6 & 1,0 & 1,0 \\
$\mathrm{DK}$ & 1,3 & 6,9 & 0,7 & 1,2 & 3,8 & 1,7 & - \\
$\mathrm{E}$ & 1,0 & 4,4 & 3,1 & 0,4 & 3,4 & 1,0 & 3,9 \\
$\mathrm{~F}$ & 0,1 & 9,4 & 2,2 & 1,7 & 3,6 & 3,0 & 2,3 \\
GR & 1,5 & 3,1 & 3,0 & 1,9 & 2,1 & 2,0 & 4,8 \\
IRL & 0,3 & 11,7 & 2,6 & 1,2 & 6,0 & 1,7 & - \\
I & 0,9 & 2,4 & 0,9 & 0,6 & 2,1 & 1,4 & 2,6 \\
L & 1,8 & 8,5 & 1,3 & 2,2 & 3,2 & 1,5 & - \\
NL & 0,3 & 4,8 & 1,3 & 1,1 & 4,0 & 0,8 & 1,4 \\
P & 2,1 & 3,8 & 7,9 & 7,5 & 5,2 & 5,9 & 5,0 \\
UK & 1,0 & 6,0 & 0,3 & 0,3 & 1,9 & 4,3 & 2,1 \\
\hline Moyenne & 1,2 & 6,3 & 2,1 & 1,6 & 3,5 & 2,1 & 3,2 \\
\hline
\end{tabular}

A partir de notre agrégation des sondages $38,39,40$ et 41 de l'Eurobaromètre.

Remarquons qu'une différence plus importante entre hommes et femmes existe en Belgique (coefficient de variation de 3,2\%) et que le type d'habitat est atypiquement très différenciateur au Portugal (coefficient de variation de 7,5\%). L'âge, que nous venons de classer parmi les critères différenciateurs de consommation des journaux télévisés, l'est tout particulièrement au Luxembourg, en Belgique, en France et surtout en Irlande (coefficients de variation respectifs de 8,$5 ; 9,1 ; 9,4$ et 11,7 pour cent). Revenus et classes sociales ne semblent pas particulièrement différenciants sauf au Portugal.

Peut-on en conclure qu'une cible européenne définies en termes d'âge ou de profession pourrait présenter quelque homogénéité ? Pas 
forcément. Entreprenons de mesurer l'homogénéité des différents groupes à travers l'Europe en calculant l'étalement des valeurs d'un même groupe dans les différents pays, c'est-à-dire en mesurant la dispersion de chaque classe de chaque critère pour chaque média à travers les douze pays (Tableau 6).

\section{Tableau 6}

Etalement inter-national des classes sociodémographiques (coefficients de variation, en pour cent)

\begin{tabular}{ll}
\hline $\begin{array}{l}\text { Etalement entre } \\
12 \text { pays }\end{array}$ & 4,5 \\
Etalement entre & \\
60 régions & 6,0 \\
Hommes & 4,3 \\
Femmes & 4,8 \\
15-24 ans & 7,6 \\
25-39 ans & 5,7 \\
40-54 ans & 4,5 \\
55 ans et + & 5,4 \\
Revenus ++ & 4,2 \\
Revenus + & 5,1 \\
Revenus - & 4,3 \\
Revenus -- & 7,0 \\
Zone rurale & 5,9 \\
Petite ville & 4,1 \\
Grande ville & 4,5 \\
Indépendant & 6,1 \\
Cadre supérieur & 5,0 \\
Autre cadre & 5,3 \\
Manuel & 6,0 \\
H./F, au foyer & 5,8 \\
Chômeurs & 5,3 \\
Cl. soc. inférieure & 5,0 \\
Cl. soc. moyenne & 3,7 \\
Cl. soc. supérieure & 5,1 \\
Moyenne écarts & \\
interrégionaux & \\
\hline
\end{tabular}

A partir de notre agrégation des sondages $38,39,40$ et 41 de l'Eurobaromètre. 
L'approche est imparfaite, puisque la dispersion des valeurs des différentes catégories sociodémographiques est mesurée sur les moyennes nationales (faute de données relatives à la dispersion dans chaque pays au sein de chaque classe).

La classe sociale moyenne présente l'étalement le plus faible. On peut donc soupçonner que, de tous les groupes envisagés (hommes, femmes, etc.), celui des individus se situant dans la classe sociale moyenne présente la plus forte homogénéité quant à la fréquence de consommation de journaux télévisés. Nettement plus, en tout cas, que les 15-24 ans et les revenus les plus faibles pour qui l'étalement est maximum. Hommes, revenus "++" et "-" et habitants de petites villes démontrent également quelque homogénéité.

\section{Journal télévisé européen}

Ces observations pourraient utilement alimenter la préparation de quelque ébauche de journal télévisé qui se chercherait une cible européenne. Mais entreprendre une telle aventure impliquerait de surmonter encore bien des obstacles. En dépit des prophéties de standardisation des produits et d'homogénéisation des publics ${ }^{1}$, les produits médiatiques demeurent culturellement marqués et les publics manifestent généralement une préférence prononcée pour les contenus d'origine nationale ${ }^{2}$ (ou régionale) ou, à défaut, élaborés dans un pays limitrophe et dans leur langue maternelle ${ }^{3}$. En télévision, il semble établi que les grands succès d'audience en prime time sont, en règle générale, acquis sur des programmes à forte connotation nationale ${ }^{4}$.

Les médias d'information constituent une catégorie singulière qui présente la particularité d'être soumise à un pôle de contraintes supplémentaires : quelle que soit la curiosité du public pour l'actualité

1 Jules Gritti évoque ces deux tendances dès les années 60 (J. GRITTI, Culture et techniques de masse, Tournai, Casterman, 1967, p. 31-36).

2 Voir notamment S. REGOURD, "Programmes et identité nationale", in J.-Y. CAPUL (dir.), Les médias, Cahiers français, $\mathrm{n}^{\circ} 266$, Paris, La Documentation française, 1994, p. 73; A. SREBERNY-MOHAMMADI, "The global and the local in international communications", in J. CURRAN \& M. GuREvitch (éd.), Mass media and society, Londres, Edward Arnold, 1991, p. 128.

3 J. STRAUBhaAR, "Beyond media imperialism: asymmetrical interdependence and cultural proximity", in Critical Studies in Mass Communication, vol. $8, \mathrm{n}^{\circ} 1$, Annondale, Speech Communication Association, 1991, p. 39 \& 55.

4 H. Michel, Les télévisions en Europe, Paris, Presses Universitaires de France, 1994, p. 5. 
internationale ou pour les regards étrangers sur des problèmes domestiques, les consommateurs de médias d'information manifestent un goût prononcé pour une perspective nationale sur les événements, pour une narration culturellement marquée de l'information, une domestication, pour reprendre l'expression de Gurevitch', de l'actualité. Même les agences de presse ont tendance à adapter leurs produits aux différents marchés nationaux ${ }^{2}$. Selon certaines études ${ }^{3}$, les processus de perception de l'information peuvent, eux aussi, varier d'un pays à l'autre.

Au regard de ce qui précède, nous estimons que des journaux télévisés européens voire des chaînes d'information européennes sont condamnées, pour l'heure, à se cantonner dans un nombre restreint d'expériences centrées sur des publics assez ciblés. Remarquons d'ailleurs que le succès avéré (ou du moins la persistance) notamment de $C N N$ semble avoir engendré, outre des projets transnationaux concurrents ${ }^{4}$, une série d'initiatives nationales de chaînes d'information en continu, rompant avec le préjugé selon lequel une chaîne thématique de ce type ne pouvait vivre qu'en agrégeant des fractions de publics disséminés à travers le continent ou la terre entière. Les "grands pays", et donc les vastes marchés nationaux, connurent les premières expériences de ce type. Ainsi, Sky News fut introduite au Royaume Uni en 1989, N-TV en Allemagne en 1992, LCI (dont le lancement fut articulé autour du concept "l'info, c'est local") en France et Télé-Noticias en Espagne. L’épidémie atteint même les marchés plus réduits. Pour preuve, le lancement, en Suisse romande, de Suisse 4. Ce retour vers l'échelon national constitue, à notre sens,

1 M. GuREvitch, "Comparative research on television news: problems and challenges", in American Behavioral Scientist, vol. 33, n², Newbury Park, Sage, 1989, p. 223.

2 Wilke et Rosenberger montrent à quel point les dépêches diffusées en Allemagne par Associated Press sont sélectionnées et adaptées (style, éléments de contexte) aux caractéristiques supposées de ce pays (J. WILKE \& B. RoSENBERGER, "Importing foreign news: a case study of the German service of Associated Press", in Journalism Quarterly, vol. 71, n², 1994, p. 421-432).

3 L. B. FaCORRo \& M. DE Fleur, "A cross-cultural experiment on how well audiences remember news stories from newspapers, computer, television, and radio sources", in Journalism Quarterly, vol.70, n³, 1993, p. 599.

4 Le succès, même relatif, sporadique (marqué en cas de crise) et inattendu de $C N N$ a engendré une série impressionnante de projets similaires, au premier rang desquels Euronews et BBC World, et, plus récemment encore (juillet 1996), MSNBC Cable (chaîne d'information fruit d'une alliance entre Microsoft et la chaîne américaine $N B C$ ), un projet similaire d' $A B C$, etc. 
l'amorce d'une tendance profonde et durable. Il est d'ailleurs remarquable que les leaders de l'actualité transnationale soient partiesprenantes dans bon nombre de leurs rejetons nationaux. 